\title{
Rejets thermiques en rivières et hydrobiologie Un aperçu sur l'expérience française
}

\author{
par Michel Khalanski et Raymond Gras
}

EDF, Département Environnement, BP 49, 7840 I Chatou Cedex

\section{I 圆 LE DÉFI DE LA POLLUTION THER- MIQUE}

A la fin des années 60, l'achèvement des grands aménagements hydroélectriques a conduit EDF à s'orienter presqu'exclusivement vers la construction de centrales thermiques pour faire face à l'accroissement de la demande en énergie électrique.

L'augmentation de la puissance unitaire des groupes thermiques classiques, passant de $125 \mathrm{MWe}$ à $600 \mathrm{MWe}$ et surtout nucléaires ( $900 \mathrm{MW}$ puis $1300 \mathrm{MW}$ ) permettait d'envisager d'équiper certains sites « jusqu'à $4000 \mathrm{MW}$ sur la Seine et $8000 \mathrm{MW}$ sur le Rhône »[1]. Il fallait en fait prendre en compte trois contraintes qui limitent nécessairement la capacité d'équipement d'un site.

Il s'agit en premier lieu de la disponibilité en eau de refroidissement $(t a b l .1)$. Les centrales nucléaires à eau légère évacuent à la source froide un flux de chaleur important, ce flux correspond à une puissance thermique équivalant à environ deux fois la puissance électrique. De ce fait, les centrales refroidies en circuit ouvert demandent de grandes quantités d'eau de refroidissement. Pour un échauffement de $10{ }^{\circ} \mathrm{C}$ au condenseur, il faut environ $47 \mathrm{~m}^{3} / \mathrm{s}$ pour une tranche nucléaire REP de 900 MWe et seuls les grands fleuves tels que le Rhin et le Rhône, ou le littoral marin, peuvent accueillir des sites de 2 à 6 tranches nucléaires en circuit ouvert. En équipant les circuits de tours de réfrigération il est toutefois possible de réduire considérablement les besoins en eau, jusqu'à $2 \mathrm{~m}^{3} / \mathrm{s}$ par tranche nucléaire de 1300 MWe. La chaleur est alors principalement évacuée par évaporation d'une partie de l'eau du circuit dont la condensation forme le panache qui couronne les aéroréfrigérants. Lorsque l'on réduit le prélèvement d'eau, la concentration en sels dissous dépasse la limite de solubilité des carbonates, il est nécessaire d'appliquer un traitement antitartre qui consiste en injections d'acides forts, l'eau rejetée est alors enrichie en sels dissous du fait de l'évaporation et en sulfates ou chlorures provenant de la « vaccination acide ».

En second lieu, il fallait éviter la recirculation des eaux échauffées, en particulier pour les centrales en bord de mer. Et sur les grands fleuves, où plusieurs sites étaient envisagés, il fallait tenir compte de l'échauffement résiduel provenant des sites en amont.

Tableau 1. - Besoins en eau de refroidissement et échauffements au condenseur des tranches thermiques classiques et nucléaires REP.

\begin{tabular}{|c|c|c|c|c|c|}
\hline Mode de réfrigération & Type de centrale & $\begin{array}{l}\text { Puissance unitaire } \\
\text { (MW) }\end{array}$ & $\begin{array}{l}\text { Débit prélevé } \\
\qquad\left(\mathrm{m}^{3} / \mathrm{s}\right)\end{array}$ & $\begin{array}{l}\text { Débit restitué } \\
\qquad\left(\mathrm{m}^{3} / \mathrm{s}\right)\end{array}$ & $\begin{array}{l}\text { Echauffement } \\
\left({ }^{\circ} \mathrm{C}\right)(1)\end{array}$ \\
\hline \multirow[t]{2}{*}{ Circuit ouvert } & Combustibles fossiles & $\begin{array}{l}125 \\
250 \\
600 \\
700\end{array}$ & $\begin{array}{c}5,5 \\
8 \stackrel{\text { à } 10}{22} \\
25\end{array}$ & $\begin{array}{c}5,5 \\
8 \stackrel{\text { à } 10}{22} \\
25\end{array}$ & $\begin{array}{c}7,5 \\
8 \\
9 \\
12\end{array}$ \\
\hline & Nucléaire REP & $\begin{array}{r}900 \\
1300\end{array}$ & $\begin{array}{c}42 \\
46 \text { à } 60\end{array}$ & $\begin{array}{c}42 \\
46 \text { à } 60\end{array}$ & 11 à 11 \\
\hline \multirow{2}{*}{$\begin{array}{c}\text { Circuit fermé } \\
\text { (aéroréfrigérants) }\end{array}$} & Combustibles fossiles & 250 & 0,2 & 0,1 & $<5$ à $10(3)$ \\
\hline & Nucléaire REP & $\begin{array}{r}900 \\
1300\end{array}$ & $\begin{array}{c}2 \text { à } 4,5 \\
2\end{array}$ & $\begin{array}{c}1,3 \text { à } 3,8 \\
1,2\end{array}$ & $<5$ à $10(3)$ \\
\hline
\end{tabular}

(1) Echauffement au condenseur it la puissance nominale.

(2) $11{ }^{\circ} \mathrm{C}$ en rivière, jusqu'ì $15^{\circ} \mathrm{C}$ en mer.

(3) Echauffement variable, moyenne menstelle minimum en été, maximum en hiver. 
Il fallait enfin respecter les limites thermiques imposées par la réglementation de l'époque qui se limitait alors à $30{ }^{\circ} \mathrm{C}$ au maximum dans les rejets (ou après mélange selon l'interprétation des textes), et tenir compte dans la planification d'une éventuelle évolution des contraintes réglementaires.

Il importait donc du point de vue économique comme du point de vue de la protection de l'environnement de connaître la capacité maximale d'équipement en circuit ouvert, ou fermé, de chaque site potentiel. Cet enjeu, déjà important dans la France des années 60, est devenu capital à partir du lancement du programme nucléaire en 1973.

Pour faire face au défi de la pollution thermique, il convenait pour chaque projet, de prévoir quantitativement les modifications de température des cours d'eau ou du milieu marin littoral. Il était aussi nécessaire d’acquérir des connaissances de base sur les effets écologiques de l'échauffement des eaux naturelles qui restaient encore très fragmentaires afin de répondre aux obligations de la loi sur la protection de l'environnement qui instituait les études d'impact. Cette tâche a été en grande partie assumée grâce à la conjonction de moyens et de compétences scientifiques et techniques diverses parmi lesquelles il convient de citer les équipes de la Direction des Etudes et Recherches et de la Direction de l'Equipement d'EDF, du CEMAGREF, de laboratoires universitaires, d'IFREMER et d'autres grands organismes nationaux de recherche.

\section{MODELISATION THERMIQUE}

Les deux facteurs décisifs qui déterminent la puissance maximale d'équipement d'un site en rivière sont sa capacité d'absorption de chaleur et sa capacité de dissipation d'énergie thermique restituée à la source froide.

- la capacité d'absorption de chaleur dépend du débit de la rivière et de la température de l'eau à l'entrée du tronçon,

- la capacité de dissipation d'énergie thermique dépend des conditions d'écoulement de l'eau dans le tronçon considéré, des conditions météorologiques et des conditions d'échanges d'énergie entre l'eau et l'atmosphère.

Sur ces bases, et à partir d'un faisceau d'études [1], [2], [3], [4], un modèle numérique simulant le régime thermique naturel et perturbé des grands cours d'eau a été développé à la Direction des Etudes et Recherches d'EDF.

Ce modèle a été notamment appliqué aux projets d'équipements de la Loire et du Rhône afin d'optimiser les équipements en circuit ouvert et en circuit fermé sur ces grands fleuves.

Des modèles numériques bidimensionnels et tridimensionnels ont été développés par le Laboratoire National d'Hydraulique pour représenter le mélange de l'eau échauffée avec l'eau de la rivière dans le champ proche des rejets [5].

\section{LES CONSÉQUENCES HYDROBIO- LOGIQUES DE L'ÉCHAUFFEMENT DES EAUX}

De 1962 à 1978, un groupe d'experts a été chargé par EDF d'évaluer les conséquences écologiques de l'échauffement des eaux. Les travaux réalisés sous l'égide de ce groupe, le Comité Scientifique de Montereau, concernent la plupart des disciplines de l'écologie appliquée aux milieux aquatiques: physico-chimie des eaux courantes, écophysiologie, microbiologie, structure des peuplements d'invertébrés et de poissons. Ces travaux ont été présentés synthétiquement dans un numéro de la revue du Comité [6].
Depuis 1979, les investigations se sont poursuivies sur le site du Bugey où le groupement de Lyon du CEMAGREF a appliqué les méthodes mises au point au laboratoire d'hydrobiologie installé auparavant à la centrale thermique de Montereau. Nous allons tenter de donner un aperç des principaux résultats acquis à l'occasion d'études particulières sur les effets de l'échauffement des eatux douces.

Enfin, les programmes de surveillance physico-chimique et hydrobiologique réalisés sur chaque site nucléaire depuis sa mise en service industrielle apportent une moisson de données irremplaçables sur l'évolution écologique à long terme des cours d'eau. Les arrêtés préfectoraux d'autorisation de rejet d'eau imposent de réaliser un contrôle de l'environnement des centrales nucléaires dont le programme est établi à la diligence de l'Administration chargée de la Police des Eaux (Services de la Navigation, Ports Autonomes) et sous l'autorité du Délégué de Bassin. Ce programme vise à assurer une surveillance de l'environnement aquatique susceptible d'être affecté par les rejets liquides non radioactifs des centrales nucléaires. Il est défini pour chaque site pour une durée de quelques années et doit être réajusté à son expiration en accord avec les parties intéressées. En complément aux données hydrobiologiques recueillies par des laboratoires spécialisés avec une périodicité dépendant de la méthode employée, chaque site est équipé d'automates fournissant en continu une valeur des quatre grandeurs fondamentales de qualité d'eau: température, oxygène dissous, $\mathrm{pH}$ et conductivité, intégrée sur un pas de temps horaire.

\subsection{Microbiologie}

Dans certaines limites, l'échauffement de l'eau augmente le métabolisme des micro-organismes et, en l'absence d'autres facteurs limitant, conduit à l'accroissement des populations de microbes adaptés à la vie aquatique, qu'il s'agisse d'algues planctoniques, de bactéries ou de protozooaires. On a ainsi observé des développements importants de bactéries filamenteuses dans le canal de rejet de la centrale de Montereau pendant l'hiver au moment où les sucreries apportent des matières organiques dans l'eau de Seine [6].

Le développement de germes pathogènes en eau échauffée peut poser un problème de santé publique, c'est pourquoi des études particulières ont été réalisées sur ce thème.

En accord avec les données de la littérature scientifique, on a montré que l'élévation locale de la température du Rhône au niveau du site du Bugey n'entraîne pas d'augmentation d'abondance des bactéries indicatrices de contamination fécale (coliformes et streptocoques), leur nombre tend au contraire à décroître en eau échauffée [7]. Sur le même site, une étude des mycobactéries a abouti aux mêmes conclusions [8]. En Moselle, sur le site de la centrale de La Maxe, la présence de salmonelles s'explique par une pollution venant de l'amont, l'échauffement de l'eau de la lagune induit vraisemblablement la sélection de certaines souches sans favoriser les plus pathogènes [9]. En fait, seuls quelques germes thermophiles sont susceptibles de proliférer dans les milieux aquatiques échauffés, c'est le cas des légionelles et de certaines amibes libres [10]. Des études approfondies ont été réalisées depuis une quinzaine d'années à l'initiative d'EDF en vue de mieux connaître l'abondance des amibes pathogènes dans les eaux échauffées et d'évaluer le risque sanitaire qu'elles représentent [11].

\subsection{Qualité des eaux, plancton}

Ainsi qu'il a été indiqué plus haut, l'echaufement influe principalement sur la qualité des eaux par le biaks des processus de dégradation des matières organiques por les bacté 
ries aérobies et, par voie de conséquences, a une incidence sur le bilan d'oxygène dissous du cours d'eau. L'autoépuration bactérienne constitue un terme important de consommation d'oxygène dans les rivières qui reçoivent des effluents organiques, toutefois, les mesures en continu d'oxygène dissous réalisées dès 1974 sur le site de la centrale Porcheville, à l'aval de Paris, ont mis en évidence l'importance capitale de la photosynthèse et de la respiration des algues planctoniques dans le bilan d'oxygène dissous du fleuve. Il importait de connaître la réponse des algues à la température, c'est pourquoi l'optimum thermique de la production d'oxygène photosynthétique a été déterminé en Seine, il se situe à 25$26^{\circ} \mathrm{C}$ et l'activité photosynthétique décroît significativement à partir de $28-30^{\circ} \mathrm{C}$ [12].

L'impact de l'échauffement sur la qualité de l'eau dans un cours d'eau « eutrophisé », la Loire moyenne, a été étudié à l'aide du modèle numérique Eutroloire [13]. Ce modèle calcule la température de l'eau, la concentration en phosphore (élément limitant) et la biomasse du plancton végétal. Dans les conditions de deux années différentes sur les plans hydrologiques et climatiques l'impact de la centrale nucléaire Saint-Laurent A $\left(50 \mathrm{~m}^{3} / \mathrm{s}\right.$ échauffés de $10{ }^{\circ} \mathrm{C}$ à pleine charge) est de quelques pourcents de la valeur de référence en biomasse du phytoplancton et l'incidence sur l'oxygène dissous n'excède pas $0,1 \mathrm{mg} / \mathrm{l}$ en moyenne.

Les mesures de chlorophylle a planctonique effectuées de 1979 à 1994 par le CEMAGREF sur des stations positionnées en amont et en aval du rejet (fig. I) montrent que l'impact du centre nucléaire du Bugey n'est pas perceptible sur la biomasse phytoplanctonique. La longue série de mesures met cependant en évidence trois périodes distinctes : au début, la chlorophylle $a$ planctonique reste inférieure à $10 \mu \mathrm{g} / \mathrm{l}$, à partir de 1985 et jusqu'en 1990, l'amplitude des variations saisonnières s'accrôt : le maximum relevé se situe entre $15 \mu \mathrm{g} / \mathrm{l}$ et $50 \mu \mathrm{g} / \mathrm{l}$, enfin, des quatre dernières années, les concentrations en chlorophylle $a$ ont retrouvé leur amplitude annuelle initiale. Un suivi à long terme de la biomasse planctonique sur un réseau de quatre stations échantillonnées cinq fois par an a permis de détecter un changement important d'une des grandeurs servant à mesurer le niveau trophique de l'écosystème. Ce fait illustre l'intérêt des programmes de mesures sur le long terme qui permettent de détecter des changements significatifs, en l'occurrence indépendants du fonctionnement des centrales.

\subsection{Invertébrés benthiques}

Nous prendrons deux exemples relatifs à un site en circuit ouvert: le Rhône au Bugey et à un site en circuit fermé : la Loire à Dampierre-en-Burly.

Au Bugey, le peuplement de macroinvertébrés est échantillonné sur les fonds en quatre stations à l'amont et à l'aval du centre nucléaire depuis 1980, trois à quatre fois par an. Les trois stations de l'aval sont différemment soumises à l'influence des rejets : celle située dans le rejet lui-même est soumise à un échauffement permanent d'environ $10^{\circ} \mathrm{C}$ et des conditions de courant particulières, celle située à l'aval en rive droite subit un échauffement moyen de $5^{\circ} \mathrm{C}$ tandis que l'échauffement moyen reste d'environ $0,5^{\circ} \mathrm{C}$ sur celle de la rive gauche.

La synthèse réalisée récemment par Roger et Ginot [14] à partir des données collectées pendant 13 ans met en évidence la grande variabilité des indices de qualité biologique fondés sur les invertébrés (IQBG et IQBP) et de la variété taxonomique. Les indices IQBG varient entre 5/20 et 17/20 sur l'ensemble de la période. Ils présentent des fluctuations saisonnières qui apparaissent aussi sur la chronique du nombre de taxons inventoriés.

Dans ce contexte fluctuant, l'impact du centre nucléaire du Bugey entre amont et aval se manifeste par ane baisse du nombre de taxons à la station rejet et à la station aval rive droite (fig. 2), l'indice de qualité biologique est aussi plus bas dans le rejet. A la station aval en rive gauche, très peu échauffée, ces effets ne sont pas observés. C'est une analyse faunistique globale, c'est-à-dire portant sur l'ensemble des 138 taxons identifiés sur le secteur, conjointement à des études relatives à des groupes particuliers [15], qui a permis de caractériser plus précisément les modifications de la structure biocénotique en rive droite du Rhône. Cette analyse montre que la plupart des taxons communs sont en fait affectés à la station échauffée de $5^{\circ} \mathrm{C}$, soit qu'ils soient favorisés (plus abondants) ou défavorisés (moins abondants) ou que leur cycle biologique soit perturbé. Une étude particulière réalisée sur la crevette d'eau douce - le gammare - a permis de dresser un profil thermique de l'espèce dans la zone échauffée [16] : au printemps la reproduction est favorisée par des températures d'environ $15^{\circ} \mathrm{C}$, l'abondance diminue dès que la température atteint $20^{\circ} \mathrm{C}$, la zone est désertée au-delà de $25^{\circ} \mathrm{C}$.

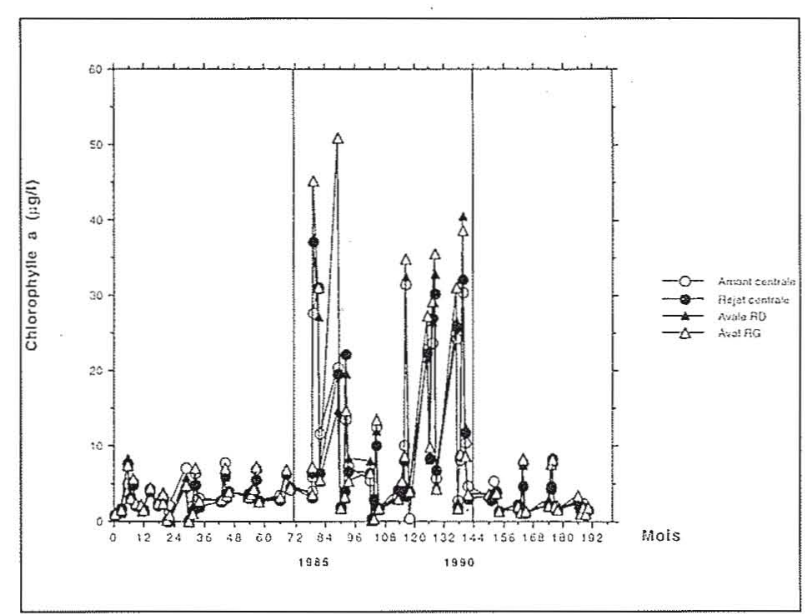

1. Evolution de la biomasse du phytoplancton mesurée dans le Rhône au Bugey de 1979 à 1984. Données du CEMAGREF provenant des rapports de surveillance de l'environnement du CNPE du Bugey.

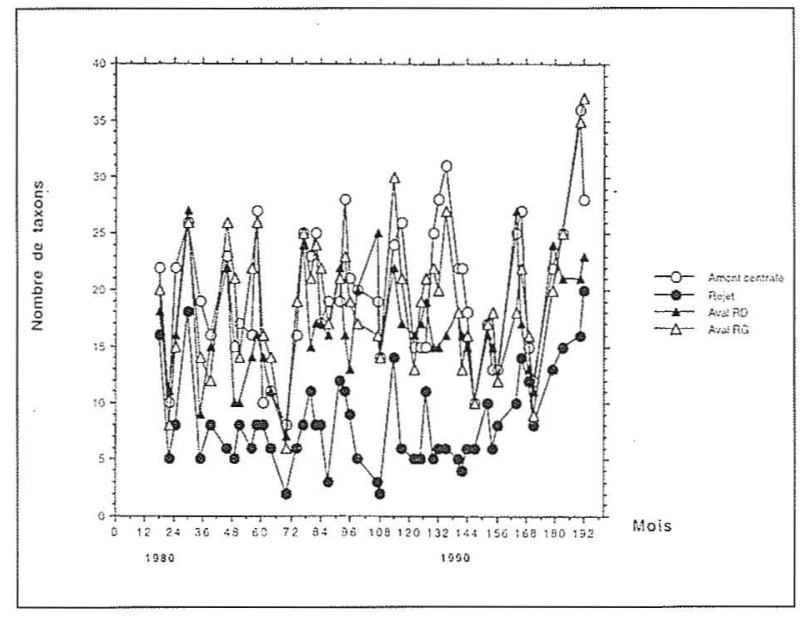

2. Variété des inyertébrés benthiques (nombre de taxons) à quatre stations en amont et aval du CNPE du Bugey de 1980 à 1994. Données du CEMAGREF provenant des rapports de surveillance de l'environnement du CNPE du Bugey. 
A Dampierre-en-Burly, trois secteurs, un en amont et deux en aval du centre nucléaire, sont échantillonnés quatre fois par an depuis 1979 par une équipe de l'Université de Clermont-Ferrand selon la méthodologie des IQBG. La synthèse des données collectées de 1979 à 1993 a été faite sous la direction de $\mathrm{N}$. Lair [17]. Contrairement aux observations faites au Bugey, l'indice et la variété taxonomique ne chute pas systématiquement entre l'amont et l'aval immédiat dusite; on note en revanche une discontinuité sur la série chronologique des indices IQBG (fig. 3) : l'amplitude des variations saisonnières de l'indice, qui atteint 11 points entre 7/20 et $18 / 20$ de 1979 à 1986 , reste ensuite limitée à 6 points, entre $8 / 20$ et $14 / 20$

Sur l'ensemble de la période, la variété n'a pas baissé, les moyenne annuelles montrent même une tendance à l'augmentation (fig. 4). La chute des valeurs maximales annuelles de l'indice est mise en rapport, par les auteurs de la synthèse des données, avec la chute de diversité du groupe des

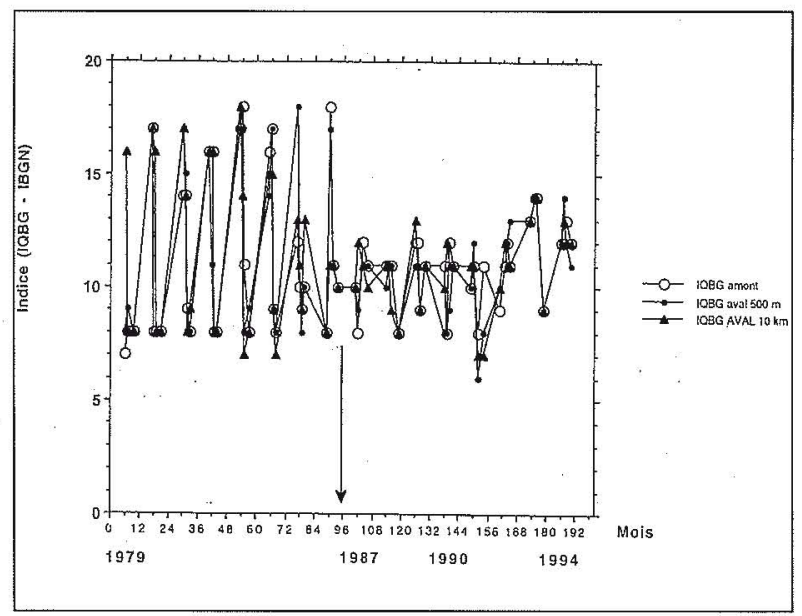

3. Indices de qualité biologique fondés sur les invertébrés benthiques (IQBG de 1979 à 1993, IBGN en 1994) mesurés en trois stations sur la Loire en amont et aval du CNPE de Dampierre-en-Burly. Données de l'Université de Clermont-Ferrand provenant des rapports de surveillance de l'environnement du CNPE de Dampierre-en-Burly.

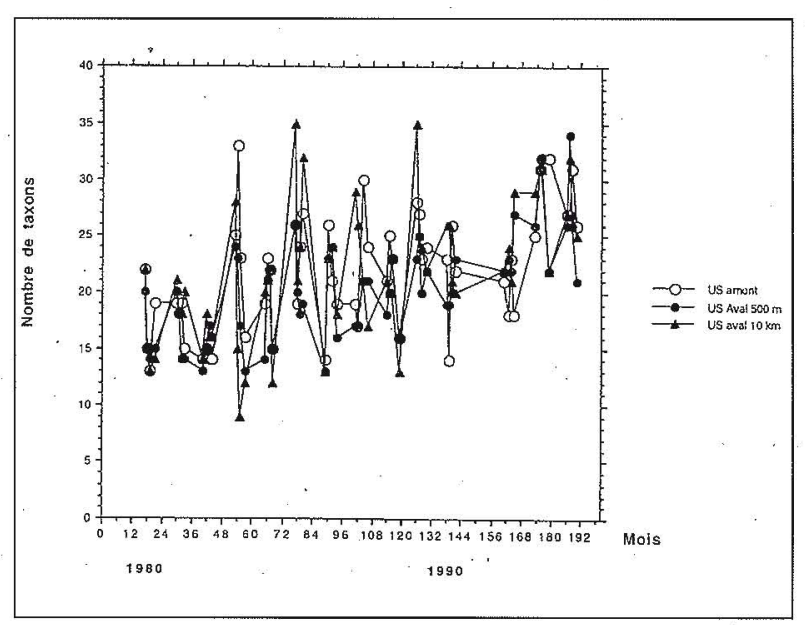

4. Variété des invertébrés benthiques nombre de taxons) à trois stations en Loire en amont et aval du CNPE de Dampierre-en-Burly de 1980 à 1994. Données de l'Université de Clermont-Ferrand provenant des rapports de surveillance de l'environnement du CNPE de Dampierre-en-Burly. insectes Ephèméroptères à partir de 1987 et une augmentation de la diversité des Trichoptères.

Le changement concerne également le peuplement d'algues du phytoplancton : à partir de 1987, les chlorophycées deviennent plus abondantes tandis que les diatomées du genre Cyclotella régressent. Ces éléments conduisent à diagnostiquer une baisse de qualité biologique à partir de 1987 sans que les causes soient pour autant identifiées.

Contrairement au site du Bugey, le faible échauffement à l'aval de ce site équipé de tours de refroidissements (quelques dixièmes de degré), explique la difficulté de mettre en évidence un impact sur les peuplements d'invertébrés benthiques. L'interprétation de certains écarts entre amont et aval du site tient compte des conditions hydrauliques locales, notamment de la création d'un seuil en Loire au niveau du site.

\subsection{Poissons}

Les poissons ont une réponse comportementale d'attraction ou de répulsion qui les fait rechercher ou fuir les parties du fleuve échauffées. Ils manifestent une préférence thermique variable selon l'espèce, l'âge des individus et la saison. Les pêches électriques standardisées mises au point par le CEMAGREF en Seine à Montereau et appliquées sur le Rhône au Bugey ont permis de mieux connaître le comportement des poissons en présence d'un gradient thermique. Ces deux cours d'eau se distinguent clairement par leur régime thermique. La Seine à Montereau est un cours d'eau de plaine relativement chaud ayant naturellement un peuplement de poissons dominé par des Cyprinidés d'eau calme; le Haut-Rhône au Bugey est un cours d'eau froid, rapide, dont le peuplement de poissons comporte des Salmonidés (truite fario et ombre) et des Cyprinidés d'eau vive.

Les pêches pratiquées dans le canal de rejet de la centrale de Montereau en 1972-1973 [18] puis de 1976 à 1980 [19] ont mis en évidence des mouvements de poissons entre le canal échauffé de $8^{\circ} \mathrm{C}$ et la Seine. Toutes espèces confondues, les effectifs capturés dans le canal étaient minimaux en hiver $\left(10^{\circ} \mathrm{C}\right.$ à $\left.12^{\circ} \mathrm{C}\right)$ et en été pour des températures supérieures à $26^{\circ} \mathrm{C}$ (fig. 5 ). Trois groupes d'espèces ont été définis à partir d'un traitement statistique des données :

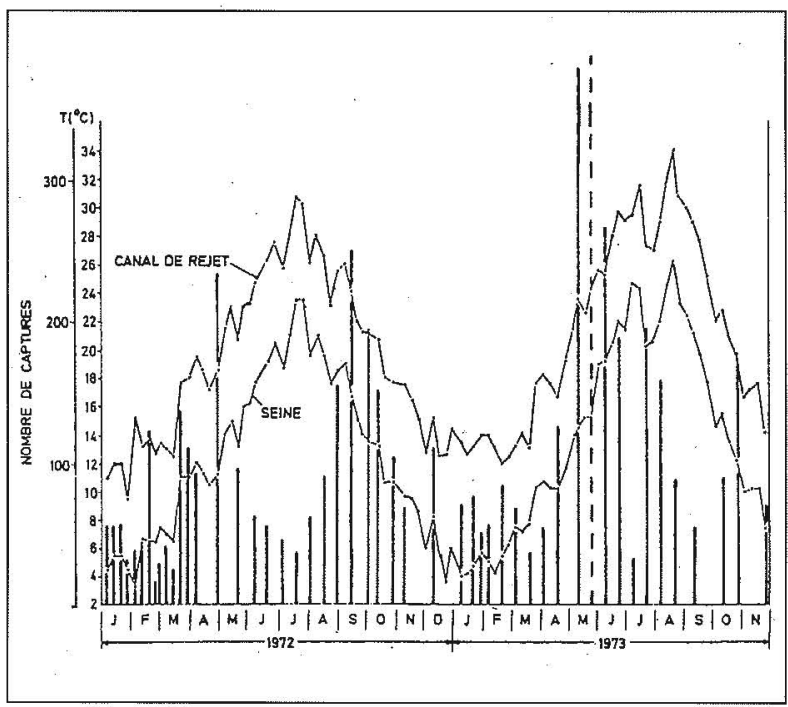

5. Evolution comparée de la température en Seine et dans le canal de rejet de la centrale de Montereau et des effectifs de poissons capturés dans le canal de rejet de janvier 1972 à novembre 1973. Emprunté à Leynaud et Allardi [18]. 
-...- les espèces pêchées plus fréquemment en dehors du canal de rejet comme le chevaine et la perche,

- Les espèces les plus abondantes dans le canal de rejet comme le poisson-chat, la tanche, la brème, la carpe, l'ablette et deux espèces thermophiles: le black-bass et la perche-soleil.

- les espèces « ubiquistes » trouvées en toutes stations comme le gardon, le brochet et le sandre.

Au Bugey, le traitement des pêches effectuées en 8 stations entre 1979 et 1992 [14] montre également un effet de la température sur les captures de poissons : la biomasse chute aux stations échauffées au-delà de $24{ }^{\circ} \mathrm{C}-25^{\circ} \mathrm{C}$ (fig. 6); ce sont les individus les plus gros qui sortent de la zone échauffée.

Au-delà de ce premier seuil thermique le peuplement s'appauvrit en adultes de truites, vandoises, barbeaux, alors que les alevins de toutes espèces continuent à fréquenter les eaux échauffées. Le second seuil thermique se situe à $29^{\circ} \mathrm{C}$, les juvéniles désertent la zone échauffée entrainant une chute brutale du nombre d'espèces.

Sur les sites en circuit fermé, les faibles échauffements relevés à l'aval des centrales n'induisent pas de déplacements liés à la température de l'eau.

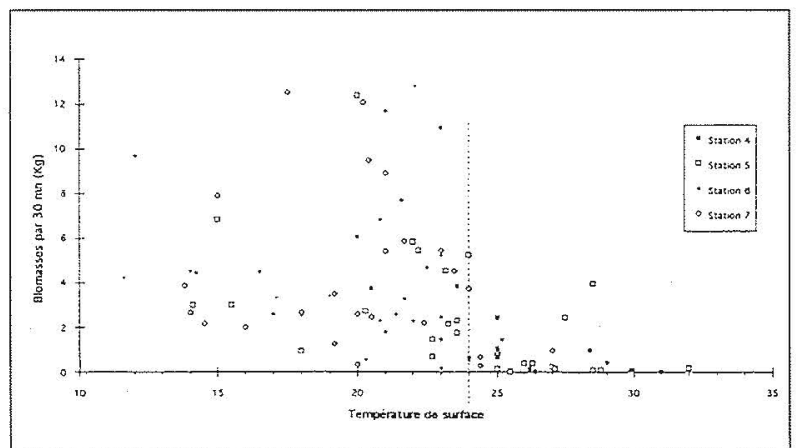

6. Biomasse de poissons récoltés par pêche électrique standardisée aux stations échauffées sur le site du CNPE du Bugey en fonction de la température de l'eau au moment des pêches. Emprunté à Roger et Ginot [14].

\subsection{Le facteur thermique dans la typologie biologique des eaux courantes et les contraintes réglementaires}

Les travaux de J. Verneaux [20] ont permis de dégager, à partir d'une analyse statistique, la structure biologique globale de l'écosystème fluvial non perturbé, et de relier cette structure à des caractéristiques morphologiques, trophiques et thermiques.

Les associations d'espèces ( 9 groupes biocénotiques) se succèdent d'amont en aval, les 3 paramètres explicatifs évoluant le long du cours d'eau.

La température intervient en tant que «température des trente jours les plus chauds de l'année ».

A partir de ce modèle structurel, on a pu déduire qu'un échauffement moyen de $1,8^{\circ} \mathrm{C}$ sur les 30 jours les plus chauds de l'année ou de $3{ }^{\circ} \mathrm{C}$ sur les 15 jours du maximum thermique, correspond théoriquement à un changement de niveau typologique, ces grandeurs pouvant être appliquées comme limites d'échauffement [21].

Les limites d'échauffement imposées dans les autorisations de prise et rejet des Centres Nucléaires de Production d'Electricité sont en partie fondées sur ces travaux et sur la directive européenne du 18 juillet 1978 relative à la qualité des eaux douces aptes à la vie des poissons. Les limites d'échauffement et de température maximales indiquées dans l'arrêté du 19 décembre 1979 sont ainsi différentes selon que le cours d'eau est salmonicole (généralement rapide et froid) ou cyprinicole (cours d'eau de plaine) :

$$
\begin{gathered}
\text { Echauffement } \\
\text { maximum }
\end{gathered}
$$

Eaux salmonicoles $+1,5^{\circ} \mathrm{C} \quad 25^{\circ} \mathrm{C}$

Eaux cyprinicoles $+3{ }^{\circ} \mathrm{C} \quad 28{ }^{\circ} \mathrm{C}$

Sur ce canevas, les limites thermiques sont définies site par site afin de prendre en compte leur spécificité sur le plan hydroécologique, elles peuvent être modulées selon la saison en fonction du régime thermique et hydrologique du cours d'eau.

\section{HYDROBIOLOGIE ET CENTRALES THERMIQUES ; ESQUISSE D'UN BILAN ET PRÉOCCUPATIONS ACTUELLES.}

Depuis 1973, 37 tranches nucléaires représentant une puissance de $38400 \mathrm{MW}$ ont été installées sur nos cours d'eau 24 tranches REP de $900 \mathrm{MW}, 12$ tranches REP de $1300 \mathrm{MW}$ et une tranche RNR de $1200 \mathrm{MW}$. Cet équipement constitue actuellement les deux tiers du parc nucléaire en exploitation, le tiers restant $(19880 \mathrm{MW})$ est installé sur le littoral marin et en Gironde.

Malgré l'importance de cet équipement, les rejets thermiques ont progressivement diminué au cours des dernières années. Cette évolution est due d'abord à la moindre sollicitation des centrales thermiques à combustibles fossiles en circuit ouvert à mesure que les tranches nucléaires en circuit fermé étaient mises en service. L'arrêt des tranches nucléaires à Uranium Naturel Graphite Gaz, sur la Loire et le Rhône, qui étaient refroidies en circuit ouvert a également contribué à cette évolution.

Les conclusions tirées des études biologiques réalisées antérieurement à la mise en service des centrales nucléaires de grande puissance ont été pour l'essentiel confirmées par les programmes de surveillance hydroécologique des sites qui fournissent actuellement des séries de données sur une quinzaine d'années.

Comme prévu, le mode de réfrigération conditionne les modalités de l'impact.

Sur les sites en circuit ouvert, il existe une zone d'impact thermique correspondant aux canaux de rejet et à la partie du fleuve, sur la rive où s'effectue le rejet généralement dans laquelle le mélange de l'eau échauffée n'est pas effectif. Dans cette partie, les échauffements sont significatifs, ils atteignent par exemple sur 1 à $2 \mathrm{~km}+1{ }^{\circ} \mathrm{C}$ à Taval de Fessenheim sur le Rhin, $+2{ }^{\circ} \mathrm{C}$ à Tricastin et $+5{ }^{\circ} \mathrm{C}$ au Bugey sur environ $10 \mathrm{~km}$. En dehors de la température de l'eau, aucune dégradation des caractéristiques physico-chimiques n'est observée, le niveau d'oxygène dissous reste notamment satisfaisant même lors d'étiages sévères (minimum de 7,3 mg/l en septembre 1985 au Bugey).

C'est dans cette zone que se manifeste l'impact thermique sur les peuplements aquatiques. Sur le Haut-Rhône, le dépassement de la température seuil de $25^{\circ} \mathrm{C}$ pendant quelques dizaines de jours entrâne une baisse de la variété des invertébrés vivant sur les fonds et provoque la fuite de nombreux poissons adultes. Ces perturbations sont transitoires pour les poissons qui recolonisent la zone d'impact thermique dès que la température redescend sous le seuil critique, il en est de même pour certains invertébrés.

Sur les sites équipés d'aéroréfrigérants, la zone d'impact thermique est limitée, quand elle existe, au canal de rejet de l'eau de purge. A l'aval du rejet, généralement effectué dans la rivière par un dispositif de mélange, les échauffements mesurés sont généralement de quelques dizièmes de degrés. 
Il est clair que, dans ces conditions, aucun effet thermique n'est détectable. Contrairement à l'eau rejetée en circuit ouvert, la purge des aéroréfrigérants n'a pas la même composition chimique que l'eau prélevée : elle est plus minéralisée du fait de l'évaporation et éventuellement du traitement anti-tartre qui ajoute des sulfates ou des chlorures ; l'évaporation accroît également sa teneur en métaux.

Le recours aux indicateurs biologiques dans le cadre des programmes de surveillance des centres nucléaires s'avère particulièrement intéressant. En complément aux mesures et analyses de qualité d'eau de base, les bioindicateurs donnent une information originale sur l'état global du système aquatique. Ils mettent ainsi en évidence des évolutions écologiques indépendantes du fonctionnement des centrales thermiques, qui affectent l'ensemble du cours d'eau pendant une ou plusieurs années.

Les questions d'hydrobiologie actuellement posées par le fonctionnement des centrales thermiques concernent davantage certains rejets chimiques, notamment les apports de métaux à l'état de traces par corrosion des condenseurs en laits, que les rejets thermiques. Des études sont également poursuivies sur les germes thermophiles - bactéries et amibes - susceptibles de se développer dans des circuits de centrales:

Il faut enfin signaler les problèmes posés par la pullulation d'organismes fixés dans les canalisations alimentées en eaux de rivières ou en eau de mer. En rivières, les principales espèces rencontrées sont des coquillages (la moule zébrée et le clam asiatique), des Bryozoaires, ou des algues qui se développent dans les parties éclairées des aéroréfrigérants. Sur les sites en rivières, il faut périodiquement procéder à un nettoyage manuel des canalisations pour maintenir les circuits de refroidissement en état de fonctionnement optimum. Une procédure de chloration choc est appliquée en cas de besoin pour nettoyer les aéroréfrigérants [21], d'autres modes de traitement sont à l'étude.

Il faut signaler que les relevés faunistiques réalisés lors de la surveillance hydrobiologique permettent de détecter I'apparition de nouvelles espèces sur un secteur de cours d'eau, c'est le cas du clam asiatique signalé depuis 1989 en Garonne au niveau du Centre nucléaire de Golfech et dans le Rhône au Bugey depuis 1990.

\section{Références}

[1] Jacquet J. et Gras R. (1970). - Simulation du comportement d'un réseau hydrographique équipé d'un ensemble de centrales thermiques; application au bassin de la Seine. $\mathrm{XI}^{\mathrm{e}}$ journées de l'Hydraulique. Société Hydrotechnique de France. Question. IV, rapport 11, $10 \mathrm{p}$.

[2] JACQUeT J. et GraS R. (1970). — Les échanges d'énergie en jeu entre un plan d'eau et l'atmosphère; détermination de la capacité de réfrigération d'un plan d'eau. Société Hydrotechnique de France. Question IV, rapport 12, 9 p.

[3] Klein P. et Momal D. (1979). — Méthode d'estimation du rayonnement solaire. Sa mise en œuvre numérique. Rapport EDF/DER. HE/31-79.15.

[4] Gilbert A., Gras R. et Roult D. (1986). - Numerical computation of natural river temperatures. International Conference on Water Quality Modelling in the Inland Natural Environment. Bournemouth England. 10-13 June 1986. paper M1, pp. 457-472.

[5] Manoha B. et Nicollet G. (1987). - Le suivi thermique des centrales nucléaires en circuit ouvert. Revue Générale Nucléaire, $n^{\circ} 1$, pp. 41-45.
[6] Peres G., Leynaud G. et Khalanski M. (1979). - Centrales thermiques et Hydrobiologie : bilan des études effectuées de 1962 à 1978 sous l'égide du Comité scientifique de Montereau. Cahiers du laboratoire d'Hydrobiologie de Montereau, $\mathrm{n}^{\circ} 8,38 \mathrm{p}$.

[7] Merle G. (1980). - Analyses bactériologiques de l'eau du Rhône au voisinage de la centrale thermique du Bugey. Cahiers du laboratoire d'Hydrobiologie de Montereau, $n^{\circ} 10$, pp. 63-76.

[8] Viallier J. et Viallier G. (1983). — Le fonctionnement des centrales nucléaires modifie-t-il la flore mycobactérienne des eaux ? Cahiers du laboratoire d'Hydrobiologie de Montereau, $n^{\circ} 14$, pp. 55-60.

[9] Noel J. et SChWARTZBrod J. (1981). - Influence d'un rejet d'eau chaude sur la survie des salmonelles. Cahiers du laboratoire d'Hydrobiologie de Montereau, n 12, pp. 7-12.

[10] Delattre M. (1984). - Pollution thermique et microbes dangereux. Recherche sur leś Legionella et les Naegleria. Cahiers du laboratoire d'Hydrobiologie de Montereau, $\mathrm{n}^{\circ} 15$, pp. 25-30.

[11] Bard D. et Sicler F. (1995). - Amibes libres et santé publique. EDF-ENSP. ISBN 285952751 6, $142 \mathrm{p}$.

[12] BoRDET F. (1977). — Influence de l'échauffement de l'eau sur la photosynthèse planctonique dans un milieu pollué : la Seine à Porcheville. Thèse de Doctorat de I'Université Paris VI, $65 \mathrm{p}$.

[13] Gosse Ph. et Khalanski M. (1987). - La modélisation numérique appliquée à l'évaluation d'impact hydrobiologique. Revue Générale Nucléaire, $n^{\circ} 1$, pp. 46-51.

[14] Roger M.C., Ginot V. (1993). - Surveillance hydrobiologique du site du Bugey. Bilan de 10 annés d'étude de l'impact thermique des rejets. Rapport CEMAGREF Lyon, 41 p.

[15] Roger M.C., Faessel B. et Lafont M. (1991). - Impact thermique des effluents du centre de production nucléaire du Bugey sur les invertébrés benthiques du Rhône. Hydroécol. Appliquée. Tome 3, vol. 1. pp. 63-110.

[16] Roger M.C., FAessel B. (1989). - Effets de l'échauffement artificiel de l'eau du Rhône sur le développement et la production des Gammaridae. Hydroécol. Appliquée. Tome 1, vol. 1-2, pp. 53-83.

[17] LAIr N. (1994). - Synthèse des écologiques de la Loire de 1977 à 1992. Site de Dampierre-en-Burly. Rapport établi pour EDF CNPE de Dampierre-en-Burly. 78 p.

[18] LeYNaud G. et AlLaRdi J. (1975). - Incidence d'un rejet thermique en milieu fluvial sur les mouvements des populations ichtyologiques. Cahiers du laboratoire d'Hydrobiologie de Montereau, $n^{\circ} 2$, pp. 65-70.

[19] ZICH. (1980). - Influence du réchauffement artificiel des eaux sur les peuplements de poissons. Mémoire stagiaire CTGREF. ENSA Rennes, 100 p.

[20] Verneaux J. (1977). - Biotypologie de l'écosystème «eaux courantes». Déterminisme approché de la structure typologique. C.R. Acad. Sci. Paris, série D, pp. 675 678.

[21] VerRel J.L. (1983). - Impact hydrobiologique des centrales thermonucléaires en cours d'implantation sur le Rhône. Cahiers du Laboratoire d'Hydrobiologie de Montereau, 14, pp. 45-54.

[22] Khalanski M et Lutz Ph. (1987). - La chloration de l'eau de réfrigération. Revue Générale Nucléaire, $n^{\circ} 1$, pp. 52-58. 